\title{
Investigation of EAS cores
}

S.B. Shaulov ${ }^{1,6, a}$, P.F. Beyl ${ }^{2}$, R.U. Beysembaev ${ }^{2}$, E.A. Beysembaeva ${ }^{2}$, S.P. Bezshapov ${ }^{1}$, A.S. Borisov ${ }^{1}$, K.V. Cherdyntceva ${ }^{1}$, M.M. Chernyavsky ${ }^{1}$, A.P. Chubenko ${ }^{1}$, O.D. Dalkarov ${ }^{1,7}$, V.G. Denisova ${ }^{1}$, A.D. Erlykin ${ }^{1}$, N.V. Kabanova ${ }^{1}$, E.A. Kanevskaya ${ }^{1}$, K.A. Kotelnikov ${ }^{1}$, A.E. Morozov ${ }^{1}$, R.A. Mukhamedshin ${ }^{5}$, R.A. Nam ${ }^{2}$, N.M. Nesterova ${ }^{1}$, N.M. Nikolskaya ${ }^{1}$, V.P. Pavluchenko ${ }^{1}$, V.V. Piskal ${ }^{2}$, V.S. Puchkov ${ }^{1}$, S.E. Pyatovsky ${ }^{1}$, V.A. Ryabov ${ }^{1,7}$, T.Kh. Sadykov ${ }^{4}$, A.L. Schepetov ${ }^{1}$, M.D. Smirnova ${ }^{1}$, A.V. Stepanov ${ }^{2}$, A.V. Uryson ${ }^{1}$, Yu.N. Vavilov ${ }^{1}$, N.G. Vildanov ${ }^{2}$, L.I. Vildanova ${ }^{2}$, I.S. Zayarnaya ${ }^{1}$, J.K. Zhanceitova ${ }^{3}$, and V.V. Zhukov ${ }^{2}$

${ }^{1}$ P.N.Lebedev Physical Institute of the Russian Academy of Sciences, Moscow, Russia

${ }^{2}$ Tien-Shan High Mountain Science Station, Almaty, Kazakhstan

${ }^{3}$ Baykonurov Dgezkazgan University, Kazakhstan

${ }^{4}$ Institute for Physics and Technology, Almaty, Kazakhstan

${ }^{5}$ Institute for Nuclear Research of the Russian Academy of Sciences, Moscow, Russia

${ }^{6}$ Moscow Institute of Physics and Technology (State University),MIPT (SU), Moscow, Russia

${ }^{7}$ National Research Nuclear University MEPhI (Moscow Engineering Physics Institute), Moscow, Russia

\begin{abstract}
The development of nuclear-electromagnetic cascade models in air in the late forties have shown informational content of the study of cores of extensive air showers (EAS). These investigations were the main goal in different experiments which were carried out over many years by a variety of methods. Outcomes of such investigations obtained in the HADRON experiment using an X-ray emulsion chamber (XREC) as a core detector are considered. The $N_{e}$ spectrum of EAS associated with $\gamma$-ray families, spectra of $\gamma$-rays (hadrons) in EAS cores and the $N_{e}$ dependence of the muon number, $\left\langle N_{\mu}\right\rangle$, in EAS with $\gamma$-ray families are obtained for the first time at energies of $10^{15}-10^{17} \mathrm{eV}$ with this method. A number of new effects were observed, namely, an abnormal scaling violation in hadron spectra which are fundamentally different from model predictions, an excess of muon number in EAS associated with $\gamma$-ray families, and the penetrating component in EAS cores. It is supposed that the abnormal behavior of $\gamma$-ray spectra and $N_{e}$ dependence of the muon number are explained by the emergence of a penetrating component in the 1st PCR spectrum 'knee' range. Nuclear and astrophysical explanations of the origin of the penetrating component are discussed. The necessity of considering the contribution of a single close cosmic-ray source to explain the PCR spectrum in the knee range is noted.
\end{abstract}

\section{Introduction}

The power spectrum of the primary cosmic radiation (PCR) has two specific ranges, namely, the so-called knee at energies of $10^{15}-10^{18} \mathrm{eV}$ and the range of energies higher than $10^{19} \mathrm{eV}$ where cut off of the PCR spectrum is supposed. The analysis of data submitted in this paper belongs to the knee range.

The PCR energy spectrum has two rather sharp breaks in this range, namely, at energies of 3 and $70 \mathrm{PeV}$. The origins of these peculiarities of the spectrum are still not clear though the main break at $3 \mathrm{PeV}$ was found in 1958 [1]; evidence in favor of the second one was first observed in the nineties [2].

The almost only experimental way of solving the problem consists in determining the PCR mass composition in the knee range. Characteristics of EAS cores at mountain altitudes is most sensitive to the composition of PCR nuclei. The inclination the power spectrum of hadrons, $E_{h}^{-\kappa}$, at $\mathrm{TeV}$ energies sharply increases with the transition from primary protons to iron nuclei. In parallel, the power slope index, $\kappa$, changes

a e-mail: ser101@inbox.ru from $\kappa \simeq 1$ to $\kappa \simeq 2$. The only detector allowing to carry out such measurements is the $\mathrm{X}$-ray emulsion chamber (XREC) [3] due to its high spatial resolution $(\sim 100$ microns $)$ and its ability to measure the energies of the most energetic single hadrons in EAS cores.

The main problem of these measurements is the timing of the XREC data, that do not have a time reference, with data of the EAS-array electronic detectors. The solution of this problem [4] has permitted to carry out such measurements in the HADRON experiment.

The creation of a hybrid installation has become possible as a result of the development of EAS techniques developed in previous experiments. In the following section a short history of the development is given for example in the CR research in the P.N. Lebedev Physical Institute (LPI, or FIAN).

\section{History}

There has been more than 80 years of CR investigations in FIAN. A purposeful EAS study by the FIAN group started in the forties with the construction of the highmountain cosmic-ray station in the Pamirs initiated by D.V. 
Scobeltsyn and S.I. Vavilov [5]. A series of observational operations with Geiger counters, a cloud chamber and calorimeter have been made. N.A.Dobrotin played a major role in expanding the work at the Pamir Station. The development of an EAS model or model of nuclearelectromagnetic cascades (NEC) in the atmosphere by G.T. Zatsepin became the major result of these operations $[6,7]$.

EAS registration by ground-based installations up to now is virtually the only method to study PCR particles at energies higher than $\sim 10^{14} \mathrm{eV}$. The NEC model is a foundation of this method. Only the NEC model permits to determine such important characteristics as the primary energy and type of the primary nucleus.

The PCR nuclear composition consists of five groups of nuclei: p, He, CNO, Si and Fe. The fraction of each nuclear group is well-known at energies below $\sim 10^{14} \mathrm{eV}$ from direct measurements carried out on board satellites.

At higher energies direct data is absent, and it is possible to determine the PCR composition by means of EAS study only. The PCR spectrum is measured up to a maximum energy $\sim 10^{20} \mathrm{eV}$ but the problem of PCR composition has not yet been solved. It is a difficult problem so new observational methods are required to progress further.

In accordance with NEC models, the main information on the type of the primary nucleus is concentrated in the spectra of the most energetic hadrons located in the central area of EAS (EAS core). The size of this area is about one meter and is basically formed by nuclear interactions. The EAS periphery (hundreds meters) is mainly formed by low-energy electromagnetic processes and hadrons, and consists mainly of muons, electrons, positrons and $\gamma$ rays. These EAS components are important and used in experiment for different purposes.

The EAS periphery permits to determine the energy of the primary particle, initiating the EAS, by using the total number of charged particles (traditionally denoted $N_{e}$ or $N_{c h}$ ). The transition from $N_{e}$ to $E_{0}$ is performed by means of the EAS model.

The core area mainly gives information about the type of primary nucleus. Certainly such a division is conditional and was made with the aim to emphasize that the main difference in EAS installations relates to the type of core detector.

\section{EAS experiment}

A cloud chamber, spark chambers, calorimeters and XRECs were used as core detectors at Tien Shan in different years [8-11].

The big ionization calorimeter (BIC) at the Tien Shan station had a thickness of lead of $1000 \mathrm{~g} / \mathrm{cm}^{2}$ and an area of $36 \mathrm{~m}^{2}$. The PCR spectrum, the sharp form of its break, the connection between the energy in the core and total number of electrons $N_{e}$ have been obtained in the EAS experiment using BIC. These outcomes were obtained with active assistance of scientists from Bulgaria, Hungary and Czechoslovakia. Determination of the energy of the break (the knee) in the PCR spectrum $\left(3 \cdot 10^{15} \mathrm{eV}\right)$ has permitted to calibrate measurements of EAS energies. Furthermore an estimation of the PCR composition in the range of the spectrum break has been obtained by studying muon number fluctuations [12].
Besides, the substantial growth of the hadron absorption length (a so-called "long-flying component") [13] was found in the region of the spectrum break. The hypothesis of abnormal magnification of charm generation cross-section in a narrow forward cone of interaction was proposed to explain the long-flying component [14].

The installation consisting of a large number of spark chambers placed underground has operated for the study of pairs and groups of muons.

The accuracy of determining the arrival direction of particles $(\sim 3 \mathrm{mrad})$ has permitted to detect pairs of penetrating particles (possibly muons), created above the installation by hadrons with energies of $\sim 10 \mathrm{TeV}$ [15].

Measurements of the anisotropy of PCR arrival directions in the energy range $E_{0}=5 \cdot 10^{13}-5 \cdot 10^{14} \mathrm{eV}$ was carried out in the Russian-Hungarian experiment, CLARA [16].

A target experiment with a calorimeter and cloud chamber placed in a magnetic field was run to determine the properties of nuclear interactions. The formation of a heavy cluster in nuclear interactions, so-called fireball, has been developed at energies above $100 \mathrm{GeV}$. This model is used till now in accelerator experiments.

Research for PCR at higher energies demanded an increase in the area of the installation. For this purpose the Wilson chamber was replaced by a big two-gap spark chamber of area $6 \mathrm{~m}^{2}$, the largest one at that time [9]. As before, the installation included a calorimeter and an XREC installed under the spark chamber. Scientists of Łodz University (Poland) actively participated in this installation.

The sizes of the cloud and spark chambers were apparently insufficient for investigations in the range of the PCR spectrum "knee" $\left(E_{0} \sim 3 \cdot 10^{15} \mathrm{eV}\right)$. Installations with areas of hundreds square meters were required for this purpose. In response to a request of the Academy of Sciences the Soviet Government supported such a project: a big X-ray emulsion chamber of area of $1000 \mathrm{~m}^{2}$ was assembled at the Pamirs and started to operate in 1975.

\section{Experiment Pamir}

The use of X-ray films under layers of lead for detecting particles was proposed in 1958 by I.D. Rapoport [17] and implemented by Yu.A. Smorodin in experiments with XREC exposed on board aircraft and at mountain altitudes [11] as well as by I.V. Rakobolskaya in underground XREC experiments for muon registration [18]. These experiments demonstrated the prospects of the new method. A scheme of XREC for detecting $\gamma$-rays and hadrons is the Pamir experiment is shown in Fig. 1.

The XREC is a passive detector of high energy particles. Hadrons and $\gamma$-rays with energies of a few $\mathrm{TeV}$ generate narrow electron-photon cascades with a typical size of $\sim 100 \mu \mathrm{m}$ in lead.

Gamma-rays with $\mathrm{TeV}$ energies are generated mainly through $\pi^{0}$-decay $\left(\pi^{0} \rightarrow 2 \gamma\right)$ and create electromagnetic cascades in the upper lead block of the hadron XREC. The cascades create dark spots on X-ray films visible to the naked eye. Hadrons ( $\pi^{ \pm}$mainly) interact with the carbon (gum) target and generate $\gamma$-rays $\left(\pi^{0}\right)$ which are detected in the lower block of XREC. 


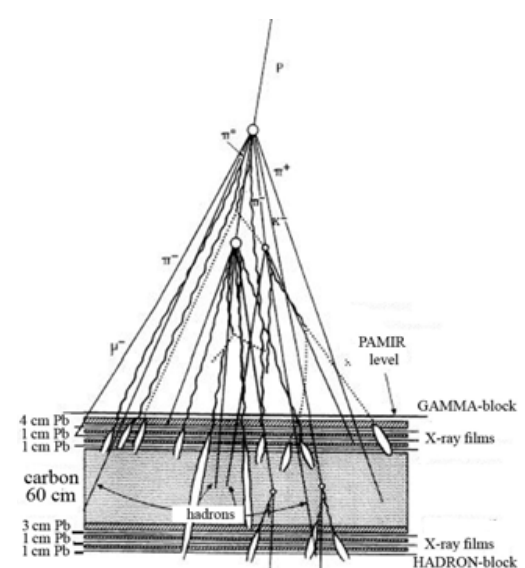

Figure 1. Scheme of XREC for detecting $\gamma$-rays and hadrons at the Pamirs.

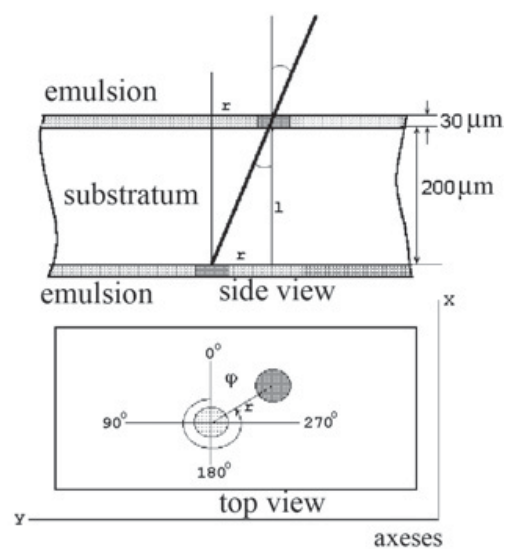

Figure 2. Scheme of angle measurements for cascades in XREC.

Measurements of the darkness of the dark spots allow the measurement of the energy and arrival angles of cascades. The energy is determined by means of a photometer and calculation procedure. Two emulsion layers on both sides of the plastic film substrate, permit to measure the shift between spots and determine the arrival angles of cascades. The scheme of angle determination in XREC is shown in Fig. 2.

The high threshold of $\gamma$-ray registration $\left(E_{\gamma} \gtrsim 1 \mathrm{TeV}\right)$ in XREC automatically selects the most energetic particles in EAS cores. The determination of arrival angles allows us to combine genetically bound cascades in gammaray - hadron families and study their energy and lateral distributions. These properties make the XREC a unique measuring tool for the study of EAS cores.

An international cooperation was established to carry out the Pamir experiment. It included more than a hundred research staff from Russia, Tajikistan, Kazakhstan, Armenia, Georgia, Poland, Japan and Brazil. The largest XRECs were assembled every year by efforts of the Pamir experiment in the Ak-Arhar gorge at an altitude of $4370 \mathrm{~m}$ a.s.1. $\left(596 \mathrm{~g} / \mathrm{cm}^{2}\right)$.

The Pamir cooperation has developed a detailed research technique for XREC usage. Some of the Russian $\mathrm{X}$-ray films were exposed at Mt.Chacaltaya in Bolivia $\left(540 \mathrm{~g} / \mathrm{cm}^{2}\right)$ [3] and on the contrary Japanese films were exposed at the Pamir experiment for cross calibration of the two techniques. As a result, some supporting evidence for the long-flying component was derived [19] and a

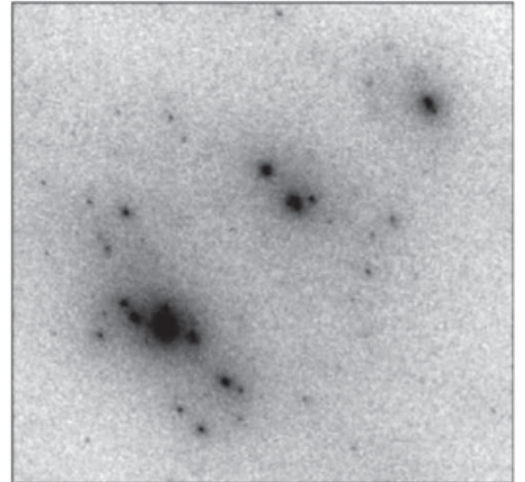

Figure 3. Aligned subgroups of high-energy particles detected in XREC.

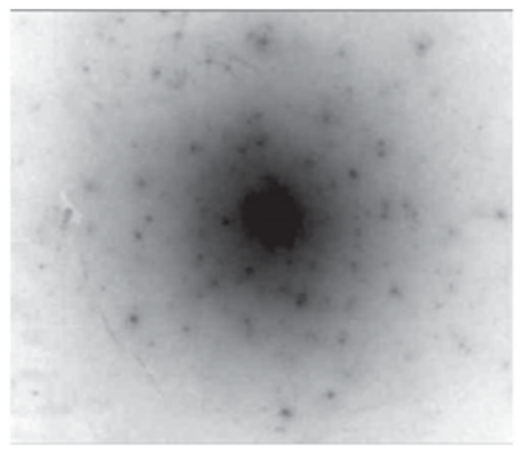

Figure 4. The halo (spot of a continuous darkening in the center of $\gamma$-ray family) with a typical size of $\sim 1 \mathrm{~cm}$.

number of new effects were observed. Interpretation of these results is prolonged till now. In particular, examples of interesting events found in the Pamir XRECs are shown in Figs. 3 and 4 which demonstrate an unusual alignment of cascade tracks along a straight line in the target plane (Fig. 3), and a so-called halo - a large dark spot at the center of the EAS core with a typical size of $\sim 1 \mathrm{~cm}$ (Fig. 4) [20,21].

These outcomes are unique as similar effects are not observed in collider experiments. Two explanations are possible. Events of such a type can be generated by protons or nuclei but fall into the forward-cone range of the interaction, which is not visible in collider experiments, or these events have an astrophysical origin and are generated by unknown particles of a new type which are present in the primary cosmic radiation.

In the early ' $80 \mathrm{~s}$ it became clear that the EAS and XREC methods separately exhausted their possibilities. On the one hand, the calorimeter technique has insufficient spatial resolution for a detailed study of the fine structure of cores. On the other hand, the XREC method does not give information on the primary particle energy. The time has come to combine these two methods.

The hybrid HADRON experiment at Tien Shan [22] has combined these two methods and continued to study EAS cores in a more appropriate way.

\section{Experiment HADRON}

The central part of the HADRON installation is schematically represented in Fig. 5.

This configuration makes it possible to determine the energy of PCR particles, the lateral distribution function 


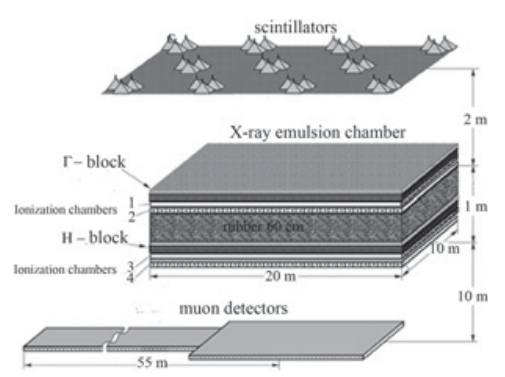

Figure 5. Scheme of the central part of the HADRON installation (top to bottom): ground-based scintillation detectors, XREC and ionization chambers, underground muon detector.

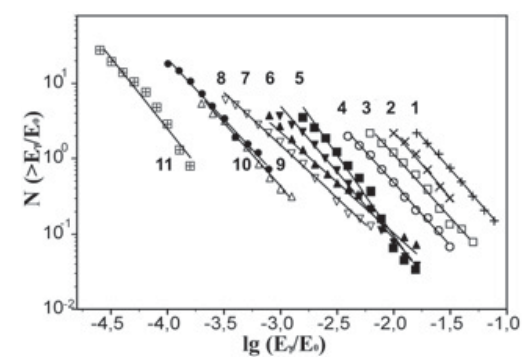

Figure 6. The experimental spectra of $\gamma$-rays energies in different intervals $N_{e}\left(E_{0}\right)$ around the break.

(LDF) of electrons and the number of muons in EAS. However, its most important part is the emulsion chamber used as a detector of the most energetic EAS-core particles. XREC makes it possible to extract unique information about the spectra of the most energetic hadrons due to the extremely high spatial resolution of XREC $(\sim 100 \mu \mathrm{m})$ and ability to measure energies of individual hadrons.

Extensive information has been obtained from measurements of EAS properties. In particular, valuable information has been derived from the analysis of EASs associated with $\gamma$-rays and/or high-energy hadrons.

EAS and XREC data were combined in this experiment, using procedures different from traditional experiments. Our group has been a pioneer in the application of a careful statistical method of combination [4]. As usual this procedure was performed by comparing the places and angles of arrival of an EAS and the associated $\gamma$-ray family. However, it was shown [4] that using only these criteria are insufficient to provide a rather small background, i.e., a low contribution of mistakenly combined events. The equation for the formation of background shows that a background value of 10-20\% can be obtained only by including several additional conditions.

- The EAS installation should work not less than $90 \%$ of calendar time during XREC exposure time (one year).

- the position of EAS axis should be determined with an accuracy not worse $10-20 \mathrm{~cm}$.

- Additional EAS selection has be done to decrease the number of EAS candidates in comparison to events in XREC. For example, one can require that a local burst in the ionization chamber with maximum ionization is above $1 \mathrm{TeV}$.

The first two requirements presented considerable challenges in the operation of the HADRON installation. Subsystems of HADRON have been doubled to support

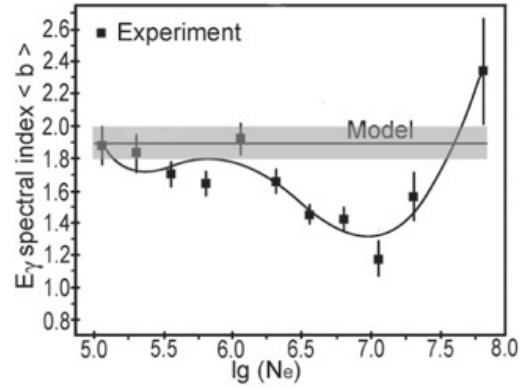

Figure 7. The $N_{e}$ dependence of spectral index $\langle b\rangle$ of the energy spectra of $\gamma$-rays, $E_{\gamma}^{-b}$.

$90 \%$ effectiveness in the case of unexpected faulty operation. Electronics of individual channels has been executed so that it could be quickly replaced. Communication circuits had photon-coupled decouplings for protection from thunderstorm and electrical noise in the conditions of high-mountainous operation and so on. The position of the EAS axis was determined by means of two layers of crosswise located ionization chambers that provided the 20-cm resolution.

The experiment $H A D R O N$ was originally planned with a view to a direct measurement of the hadron spectra in the knee energy range. Usage of XREC allowed us to select proton showers among all EASs because EASs associated with $\gamma$-ray families must be mainly generated by primary protons as predicted by all the NEC models. On the whole, for this reason different models predicted a similar result: spectra in different intervals of primary energy have close slopes, i.e., an approximate scaling has to be observed. It is a consequence of the small efficiency of heavy nuclei to generate high energy hadrons ( $\gamma$-rays). So the weighting of PCR composition behind the knee should not be affected since $\gamma$-ray-hadron families should be mainly generated by protons. Therefore any decrease of the proton fraction should only affect the intensity of events but not the form of spectra (declination). So scaling (or its weak violation) was expected because of nuclear and QCD effects associated with softer spectra of hadrons with increasing primary energy. However, expectations are not met [23].

\subsection{Abnormal scaling violation in $\gamma$-rays spectra}

The experimental spectra of $\gamma$-ray energies in different intervals of $N_{e}\left(E_{0}\right)$ are shown in Fig. 6. The numbers in the figure designate the numbers of logarithmic intervals in the range $\lg N_{e}=5.0-7.25$ through $\triangle \lg N_{e}=0.25$.

Figure 6 indicates that the declination of the spectra varies immediately behind the break of the EAS spectrum at $\lg N_{e}=6.1$, and such a correlation is unlikely to be accidental. A $N_{e}$ dependence of the spectral index $\langle b\rangle$ of the energy spectra of $\gamma$-rays, $E_{\gamma}^{-b}$, is shown in Fig. 7. It illustrates that a strong scaling violation takes place in contradiction with model predictions and this violation has an abnormal fashion. Declinations of the spectra are diminishing, i.e. the contribution of the most energetic hadrons increases and the spectra become harder. The regular character of $b\left(N_{e}\right)$ dependence is one more demonstration of a reality of observable scaling violation. Ya. Zeldovich spoke: "If experiment confirms the theory, it is pleasant. If it does not confirm, it is interesting". 


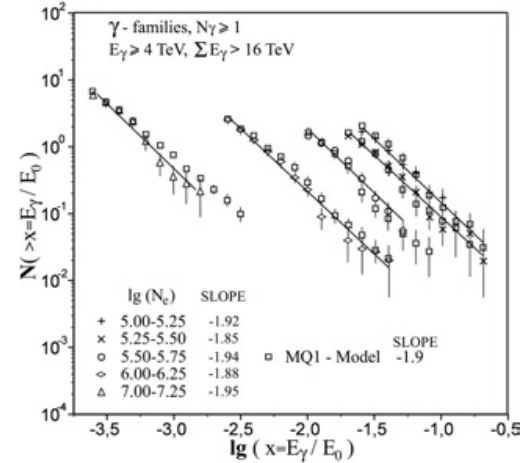

Figure 8. Observational and theoretical energy spectra of $\gamma$-rays in $N_{e}$ intervals where they approximately agree.

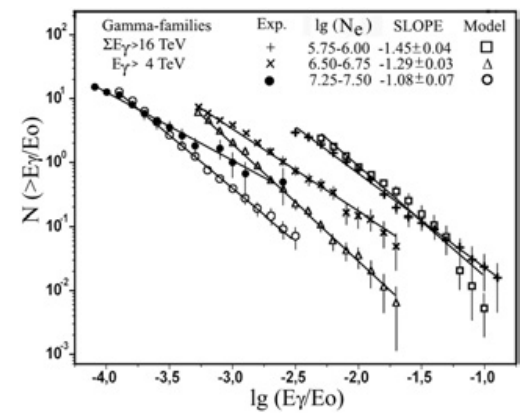

Figure 9. Observational and theoretical energy spectra of $\gamma$-rays in $N_{e}$ intervals where they differ.

A comparison between experimental and theoretical spectra of $E_{\gamma}$ in Figs. 8 and 9 shows that there are $N_{e}$ intervals where experimental and model spectra are in good agreement and where they differ considerably in slopes. Energies $E_{\gamma}$ of $\gamma$-rays are divided on primary energy $E_{0} \simeq 0.002 N_{e}$ to represent spectra in terms of the parameter $x=E_{\gamma} / E_{0}$, similar to the Feynman variable.

The results shown in Fig. 8 mean that the model correctly describes, in general terms, the experimental data. The sharp contrast between experimental and model spectra in Fig. 9 (difference exceeds $5 \sigma$ ) means that the break of CR spectra may be directly associated with some abnormal modification of CR nuclear composition.

Two explanatory variants of an experimental minimal value $b \simeq 1.2$ are possible: only light nuclei (protons) are present in the range behind the break, or an unusual new $\mathrm{CR}$ component arises here, which effectively generates $\gamma$-rays (hadrons) with hard spectra. Both explanations are exotic, but the variant with light nuclei is obviously contrary to muon data.

\subsection{Excess of muons in EAS with $\gamma$-ray families}

The $N_{e}$ dependences of the average muon number $\left\langle N_{\mu}\left(N_{e}\right)\right\rangle$ for all EAS and EAS with $\gamma$-ray families are shown in Fig. 10.

All models predict that EAS with $\gamma$-rays are mainly generated by light nuclei. Therefore $N_{\mu}$ for them should be less than average values for all EAS. This prediction is arbitrarily shown by the dotted line in Fig. 10.

The observational data appears above the average values! A prominent feature of nuclear cascades is the following, namely, light nuclei (protons mainly) generate

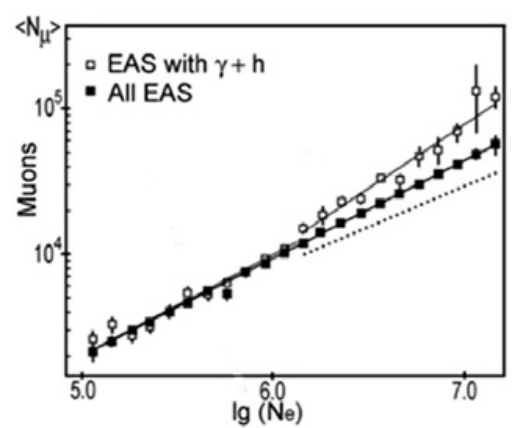

Figure 10. Experimental $N_{e}$ dependence on the average muon number $\left\langle N_{\mu}\left(N_{e}\right)\right\rangle$ : full squares -for all EAS (an inclination index $\kappa=0.7)$, empty squares -for EAS associated with $\gamma$-ray families $(\kappa=0.9)$. The dotted line is an expected dependence for EAS with $\gamma$-ray families.

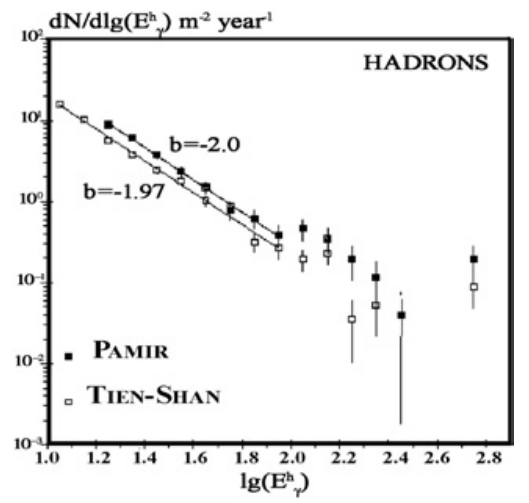

Figure 11. Comparison of hadron spectra observed with XREC at levels of the Pamirs $\left(594 \mathrm{~g} / \mathrm{cm}^{2}\right)$ and Tien Shan $\left(690 \mathrm{~g} / \mathrm{cm}^{2}\right)$.

$\gamma$-rays of high energies, but the number of muons in their EAS have to be relatively low.

On the contrary, EAS generated by heavy nuclei should contain many more muons, but virtually do not contain high-energy $\gamma$-rays. Therefore EAS with abnormal rigid $\gamma$-ray spectra and, simultaneously, with a maximal number of muons cannot be explained by traditional nuclearcascade models. It remains to speculate that the area of the break is shaped by showers of unknown origin.

This unexpected and very important result may signal the existence of a new kind of CR component, which does not consist of nuclei [24,25], or nuclear cascade developments change cardinally in such a way that cascades generated by Fe nuclei become more penetrating then proton-initiated cascades.

\subsection{The penetrating component}

The comparison of hadron spectra observed with XREC at levels of the Pamirs $\left(594 \mathrm{~g} / \mathrm{cm}^{2}\right)$ and Tien Shan $\left(690 \mathrm{~g} / \mathrm{cm}^{2}\right)$ gives an unusually large value of the absorption length: $\lambda_{a t t}=240 \pm 41 \mathrm{~g} / \mathrm{cm}^{2}$. The spectra are shown in Fig. 11.

Figure 12 shows the result of comparing energy spectra of $\gamma$-rays in $\gamma$-ray families with observed energies $\sum E_{\gamma} \geq 100 \mathrm{TeV}$.

The maximum difference of $E_{\gamma}$ spectra for levels of the Pamirs and Tien Shan does not exceed 2 but has to be $\sim 2.5$. This difference disappears with increasing $E_{\gamma}$. 


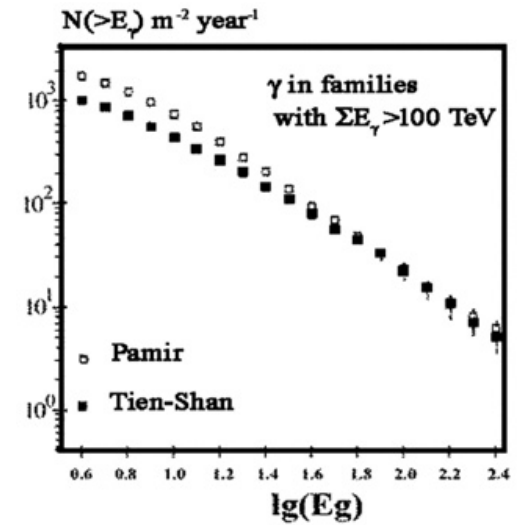

Figure 12. Energy spectra of $\gamma$-rays in $\gamma$-ray families with observed energies $\sum E_{\gamma} \geq 100 \mathrm{TeV}$.

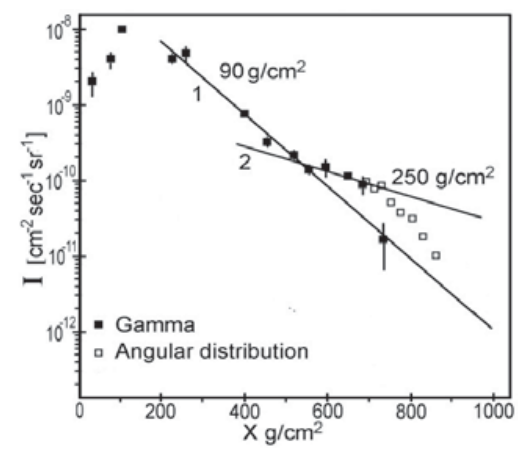

Figure 13. The absorbtion curve in an atmosphere of an electromagnetic component with $n_{\gamma} \geq 1 \mathrm{TeV}, \sum E_{\gamma} \geq 5 \mathrm{TeV}$.

The presence of a new penetrating component in CR proves to be true by the shape of the absorption curve of the electromagnetic component in the atmosphere detected by XREC shown in Fig. 13 [22]. These are results collected from different experiments using XRECs made on board balloons, airplanes and at different mountain heights of ground-based XREC [26]. Absorption is described by two exponents: with absorption length $\lambda_{a t t} \simeq 90 \mathrm{~g} / \mathrm{cm}^{2}$ (1) (as predicted by traditional nuclear-cascade models) and with a much larger value $\lambda_{a t t} \simeq 250 \mathrm{~g} / \mathrm{cm}^{2}$ (2) produced by some unknown component.

\subsection{PCR spectrum and model of a close single source}

One more important object of examination is the PCR energy spectrum. The spectrum measured in the HADRON [2] and GAMMA [28] experiments as well as in TA [29] and HiRes [30] experiments at higher energies are shown in Fig. 14. The scheme of shaping the PCR spectrum considered by a model of a single close source [31-34] is shown in Fig. 15. All spectra are multiplied by $E_{0}^{3}$ to emphasize features of the PCR spectrum.

Data of the $\gamma$-ray spectra (Fig. 7) and form of the PCR spectrum come into conflict with diffusion and accelerating models of the PCR spectrum in the area of the first knee. The model of a single close source may be added to correct this situation. It permits to represent the scheme of the PCR spectrum as the sum of a few components. The smooth spectrum 1 in Fig. 15 is explained by the radiation of many PCR sources in the Galaxy. Its declination varies

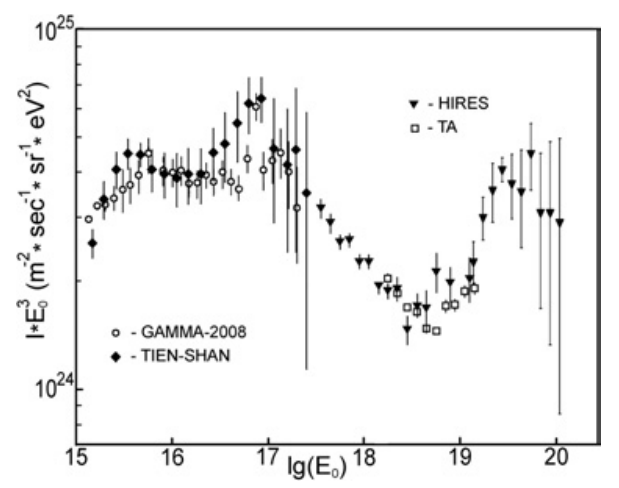

Figure 14. The PCR energy spectrum measured in HADRON, GAMMA, TA and HiRes experiments.

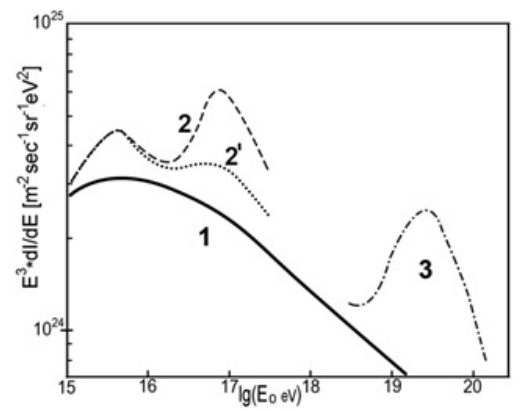

Figure 15. The scheme of the PCR spectrum formation in the model of a single close sours.

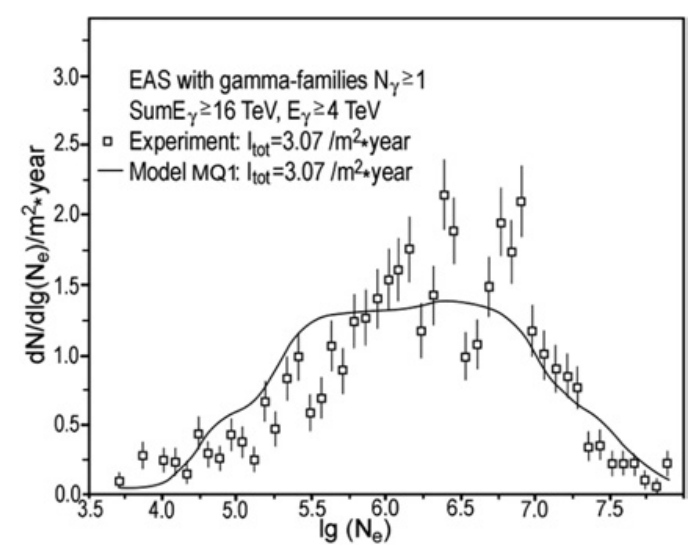

Figure 16. The $N_{e}$ spectrum of EAS associated with $\gamma$-ray families.

continuously according to diffusion models and/or the accelerating model. The spectrum 2 , which is limited by local area of energies, is shaped by a single close source. We have not yet touched on how part 3 of the PCR spectrum in Fig. 15 is shaped because it is far beyond the capabilities of our installation.

The form of spectrum 2 is represented with two variants 2 and $2^{\prime}$ because the bump at an energy of $\sim 10^{17} \mathrm{eV}$ is not observed in experiments performed at sea level.

Another argument in favor of a model of a close source follows from the shape of the experimental spectrum of EAS with $\gamma$-ray families. The experimentally obtained spectrum and a spectrum calculated using the MQ1n model (solid line) [27] is shown in Fig. 16.

The spectrum of EAS associated with $\gamma$-ray families has been detected in the experiment HADRON for the first time. It demonstrates that EAS $+\gamma$-ray family spectrum 
has a thin structure which consists of two-three peaks. The peaks are very narrow and are also located close to each other. It is possible only if the fluctuations are small enough and may be understood if there is only a single source.

What can we say about the possible position of the single close source? At first a spherical layer 200400 parsec in thickness has been defined around the sun system where this source may be located [31]. Besides, an original method to determine the direction to the source was suggested by V.P. Pavluchenko. This direction was determined by means of data of the GAMMA experiment [28]. These two results show that source may be located in the Vela constellation.

\section{Conclusions}

- EASs contain a penetrating component.

- The presence of a penetrating component can explain the anomalous violation of scaling in the spectra of $\gamma$-rays in $\gamma$-ray families associated with EASs.

- To explain the excess of muons in EASs associated with $\gamma$-ray families, we should assume a fundamental change in the characteristics of nuclear cascades in the atmosphere.

- Experimental data confirm the model of a single close source.

The origin of the penetrating component may be associated with an unusual experimental effect of the growth of the proton elastic cross section found by I.M. Dremin from the comparison data of UNK (Serpukhov) and the LHC and presented at ISVHECRI 2016.

Depending on the size of this growth, features of nuclear cascades can change dramatically. An increasing absorption length should obviously lead to the emergence of a penetrating component and to some increase of $\gamma$ ray energies at the level of observation (anomalous scaling violation). More difficult to explain is the excess of muons. In this case it is necessary to assume that due to the large number of nucleons iron nuclei generates $\gamma$-ray families with hard spectra more efficiently than a single proton. In any case, this effect is of great interest from the point of view of possible explanations of the observed effects. The situation may be clarified by model calculations of nuclear cascades with an increase in the elastic cross section of nucleons.

\section{References}

[1] Khristiansen G.B., Kulikov G.V. About the extensive air showers particles number spectrum, JETPh, 1958, 35, 3(9), p. 635-640 (in Russian)

[2] S.B. Shaulov, EAS data at the mountain level and a shape of the CR spectrum beyond the break., Nuclear Physics B (Proc. Suppl.) 122 (2003); arXiv: 1610.07427v1 [astro-ph.HE] 24 Oct. 2016

[3] PAMIR Collaboration, Investigation of nuclear interactions in the area of energies of $10^{14}-10^{17} \mathrm{eV}$ by EC method in cosmic rays (experiment "Pamir"), Trudy FIAN, 1984, 154, p. 3-141 (in Russian)

[4] S.B. Shaulov, Statistical criteria for the combination events in EXC and EAS (Exp."Hadron"). Preprint FIAN, 1987, N 245, 3-12
[5] N.A. Dobrotin. Short history of the first phase of expedition jobs on the cosmic rays in P.N.Lebedev Physical institute AS of the USSR, Moscow 1998 (in Russian)

[6] G.T. Zatsepin, Nuclear cascade process and its role in development of the EAS, DAN USSR, 1949, 67, p. 993 (in Russian)

[7] N.A. Dobrotin, G.T. Zatsepin, I.L. Rosental et al., Extenciv air showers of cosmic rays, Uspekhy Phys. Nauk, 1953, 49, p. 186 (in Russian)

[8] Guseva D.D. et al., New installation..., Izv. AN USSR, ser. phis., 1966, 30, p. 1574-1576 (in Russian)

[9] Kotelnikov K.A., Shaulov S.B., Zvonkov Ju.E., Twogap spark chamber with area $6 \mathrm{~m}^{2}$, PTE, 1977, N5, p. 66-67 (in Russian)

[10] T.P. Amineva et al., Installation for EAS study..., Trudy FIAN, 1970, 46, p. 157-176, Moscow, Nauka (in Russian)

[11] Baradzey L.T., Kanevskaya E.A., Smorodin Ju.A., Procedure of measuring of energy..., Trudy FIAN, 1970, 46, p. 200-211 (in Russian)

[12] Nikolsky S.I., Aseikin V.S., Benko D., Danilova T.V., Kabanova N.V., Stamenov J.N., Fluctuations of numbers of $\mu$-meson and depandance of number of mesons on number of particles in showers at level of mountains, Izv. AN USSR, Ser. fiz. 34, N9, p. 1955 1957, 01.1970 (in Russian)

[13] Yakovlev V.I., Long flying component: recent data and interpretation, Int. Symp. on VHE CRI. Ann Arbor. USA, 1992, p. 154

[14] Dremin I.M. et al., Monte Carlo simulations of longflying cascades in cosmic rays and leading charm at SSC, Int. Symp. on VHE CRI. Ann Arbor, USA, 1992, p. 534

[15] Basarov E.V., Beisembaev R.U., Besshapov S.P., Vavilov Yu.N., Vildanova L.I. Muon pairs observed on Tien Shan arrangement. Proc. 17-th ICRC, Paris, 7, p. 48-51; Besshapov S.P., Vavilov Yu. N. The possible role of heavy long living hadrons in muon pair creation by EAS hadrons in dense substance. Proc. 18-th ICRC, Bangalore, 1983, 5, p. 106-109

[16] D.G. Benko, G. Erdos, S.I. Nikolsky, N.M. Nesterova, V.A. Romakhin, A.P. Chubenko, A.L. Shchepetov, A. Somogyi, A. Varga, Search for sources of primary cosmic rays at energies above $0.1 \mathrm{PeV}$ at the Tien Shan, Nuclear Physics B (Proc. Suppl.), 2008, 175-176, p. 541-543, XIV ISVHECRI, 2006, Weihai, China

[17] Rapoport I.D., Photographic method of dense showers detection for the charged particles, JETF 34, 1958, p. 998-1000 (in Russian)

[18] Rakobolskaya I.V., monograph, The investigation of muons of super high energies, 1975, Izd. NAUKA, p. 3-210 (in Russian)

[19] Hlytchieva V.S., Sveshnikova L.G. et al. Anomalies of hadron attenuation at large depth of lead. // Proc. of 6 ISVHECRI. Tarbes. France. 1990, p. 184; Arisawa $\mathrm{T}$. et al. Observation of attenuation behavior of hadrons in extremely high energy cosmic ray interactions: New hadronic state?, Nucl. Phys. B 424 (1994) 241-287

[20] A.S. Borisov, Z.M. Guseva, V.G. Denisova et al., Coplanar particles emission in the process of 
the multiple births at high energies (experiment "Pamir"), Izv RAN, ser. fiz., 1994, 58, N12, p. 10-12 (in Russian)

[21] V.V. Arabkin, V.A. Borodkin, M.D. Smirnova, Yu.A. Smorodin, S.B.Shaulov. The haloes spectra of super families at the pressures $585 \mathrm{~g} / \mathrm{sm}^{2}$ and $690 \mathrm{~g} / \mathrm{sm}^{2}$ and nucleons absorption path at the energy about $10^{16} \mathrm{eV}$. 6-th ISVHECRI, Tarbe 1990 , 257-260; Pamir Collaboration. Experimental researches of the halo at superfamilies. Izv. AN USSR. Ser. fiz., 1982, 46, 9, 1790-1792 (in Russian)

[22] S.B. Shaulov, Cosmic rays about strange quark stars, monography, ISBN: 978-3-659-77433-1, LAP LAMBERT Academic Pablishin, Deutchland, 2015, p. $1-129$

[23] Arabkin V.V., Cherdyntceva K.V. et al, The abnormal behaviour of $\gamma$-ray families characteristics at primary energy $10^{16} \mathrm{eV}$, Izv. AN USSR, ser. fiz. 55, N4, p. 674-677, 1991 (in Russian)

[24] S.B. Shaulov, S.P. Bezshapov, Looking for Strange Quark Matter in Cosmic Rays, Proc. of XVII ISVHECRI 2012, European Physical Journal, Web of Conferences 52, 04010, p. 1-8 (2013)

[25] S.B. Shaulov, Hypothesis of strange quark matter in cosmic rays, Preprint FIAN, N19, p. 3-42, 2012, Moscow

[26] S.B. Shaulov et al., A peculiarity of high energy gamma-rays absorption in the atmosphere (EC data), Nuclear Physics B (Proc. Suppl.) 122 (2003)

[27] A.M. Dunaevsky, M.V. Zimin, Study of the detection of gamma-hadron families in carbon chamber,
BULLETIN de la société des sciences et des lettres de LÓDŹ, série: recherches sur les déformations, vol. XVI, 1994

[28] V.P. Pavluchenko and Collaboration GAMMA, Bulletin of the Lebedev Physics Institute, 2014, 40(5), p. 55

[29] D. Ikeda1 for the Telescope Array collaboration*: Results from the Telescope Array Experiment, Astrophys. Space Sci. Trans. 7, 257-263, doi: 10.5194/astra-7-257-2011, 2011

[30] R.U. Abbasi, T. Abu-Zayyad, M. Allen et al., First Observation of the Greisen-Zatsepin-Kuzmin Suppression, Phys. Rev. Lett. 100, 101101, doi: 10.1103/PhysRevLett.100.101101, 2008a; http:// arxiv.org/abs/astro-ph/0703099v2 15 Feb. 2008

[31] Erlykin A.D., Wolfendale A.W., A single source of cosmic rays in the range $10^{15}-10^{16} \mathrm{eV}$, J. Phys. G: Nucl. Part. Phys. 23 (1997) 979-989

[32] Erlykin A.D., Wolfendale A.W., High energy cosmic ray mass spectroscopy I. Status and prospects., Astroparticle Physics 7 (1997) 1-13

[33] A.D. Erlykin, A.W. Wolfendale, High energy cosmic ray mass spectroscopy II: Masses in the range $10^{14}-10^{17}$ eV., Astroparticle Physics 7 (1997) 203-211

[34] A.D. Erlykin, M. Lipski, A.W. Wolfendale, High energy cosmic ray spectroscopy. IV. The evidence from direct observations at lower energies and directional anisotropies, Astroparticle Physics 8 (1998) 283-292 